\title{
Goals of the SNMMI-TS Quality Committee
}

\author{
April Mann
}

Hartford Healthcare Corporation, Hartford, Connecticut

$\mathbf{A}_{\text {s the health-care environment continues to change into }}$ a quality-focused system centered on evidence-based outcomes, the nuclear medicine community continues to face external pressures, including scrutiny about patient radiation exposure, a continued perception of overuse of testing, a decrease in reimbursement, and a decrease in technologist jobs because of the nation's economy. These changes in health care, coupled with additional external pressures, will play a significant role in molding the future of nuclear medicine. To remain successful in this ever-changing health-care environment, SNMMI-TS will need to focus on, first, raising awareness in the health-care community about the value of nuclear medicine procedures in patient care and treatment and, second, ensuring that technologists have the training, competence, and credentials to perform these procedures in the new hybrid multimodality environment. It is also imperative that SNMMI-TS collaborate and build relationships with all stakeholders within the community to ensure widespread acceptance and credibility.

Quality systems that focus on evidence-based patient outcomes and satisfaction are being used by the Centers for Medicare and Medicaid Services for reimbursement and education-from competency to recertification. Additionally, several states have mandated reporting of specific measures, and private payers are using other, similar, quality initiatives as an efficient way to cut costs and drive down reimbursement. Although the strong emphasis on quality is relatively new to most health-care professionals, almost $12 \mathrm{y}$ ago the Institute of Medicine published the 6 elements every healthcare system should encompass in order to achieve high-quality patient care (1): safety (avoiding injuries to patients from the care that is intended to help them), effectiveness (providing services based on scientific knowledge to all who can benefit, and refraining from providing services to those not likely to benefit), patient-centeredness (providing care that is respectful of and responsive to individual patient preferences, needs, and values, and ensuring that patient values guide all clinical decisions), timeliness (reducing waits and sometimes-harmful delays both for those who receive care and for those who give

\footnotetext{
Received Nov. 6, 2017; revision accepted Dec. 7, 2017.

For correspondence or reprints contact: April Mann, Hartford Healthcare Corporation, 80 Seymour St., JB722 Suite M, Hartford, CT 06102.

E-mail: april.mann@hhchealth.org

Published online Dec. 22, 2017.

COPYRIGHT (c) 2018 by the Society of Nuclear Medicine and Molecular Imaging. DOI: $10.2967 /$ jnmt.117.204925
}

care), efficiency (avoiding waste, including waste of equipment, supplies, ideas, or energy), and equitability (providing care that does not vary in quality because of personal characteristics such as sex, ethnicity, geographic location, and socioeconomic status).

One of the most important areas within the health-care field is the understanding and implementation of the transition to evidence-based outcomes and quality measures to drive high-level performance. The technologist of today must be skilled in assessing patients, applying safety practices for staff and patients, and educating and instructing patients. In addition, the technologist must be able to evaluate images for technical issues, demonstration of anatomy, and overall quality. Technologists must also be able to triage and understand the disease processes being evaluated and how nuclear medicine diagnostic examinations can drive care and treatment.

To create programs addressing the new focus on quality in nuclear medicine, an SNMMI-TS quality committee in collaboration with the McKinley Advisors firm developed a survey investigating how quality is viewed, measured, and learned by technologists in the nuclear medicine field today. The survey was administered to 27,989 technologists from July 28 to August 18, 2016. SNMMI partnered with the ARRT and the NMTCB to compile the distribution list, enabling the survey to reach a broader audience. The survey garnered 4,007 responses, for a $14.3 \%$ response rate. The full results were published in the June 2017 JNMT.

The quality committee developed goals based on the survey to ensure technologists are prepared for the many clinical challenges they will face, and a 5-y plan toward achieving these goals was put into place. The objectives are to enhance quality through education and to use advocacy, leadership, public relations, outreach, and collaboration to help those outside the profession become more aware of the need for quality. Such objectives will arm technologists with the tools not only to provide patients with a good experience but also to demonstrate the high quality and safety of nuclear medicine procedures.

The first $2 \mathrm{y}$ of the 5-y plan focus on identifying avenues of collaboration among stakeholders, securing funding for ongoing and future initiatives, creating and implementing quality-education programs, developing an awareness campaign, and demonstrating quality as it relates to the continuum of care. The last $3 \mathrm{y}$ focus on quality-related continuing education initiatives; collaboration with external stakeholders; 
collaboration with organizations such as JRCNMT, IAC, and ACR; integration of quality awareness into standard educational curricula; collaboration with the SNMMI-TS advocacy committee to incorporate quality-program monitoring into the scope of practice for technologists; collaboration with ARRT and NMTCB to incorporate quality-program monitoring into continued-certification requirements; and improvement of awareness and outreach.

These goals will also support the focus on improved quality, value, and safety within the SNMMI-TS strategic plan. The specific goals of the plan-to demonstrate the quality, value, and safety of nuclear medicine and molecular imaging - are supported by the following goals of the 5-y plan: improving the quality of nuclear medicine and molecular imaging test results; developing quality measures showing the value of nuclear medicine in the continuum of patient care; implementing and educating technologists about dose optimization, safety standards, and best practices; and defining areas in which collaboration with stakeholders is key to improved quality, value, and safety in nuclear medicine.

\section{REFERENCE}

1. Institute of Medicine. Crossing the Quality Chasm: A New Health System for the 21st Century. Washington, DC: National Academies Press; 2001. 